\title{
Dynamics Analysis of Castor Hulling in the Process of Air-and-Screen Cleaning
}

\author{
Ruitao Gao, Yuhua Cao, Liangliang Yao and Hong Jin \\ Key Laboratory of Key Technology on Agricultural Machine and Equipment, Ministry and Education, South China Agricultural University, \\ Guangzhou 510642, China
}

\begin{abstract}
The air-and-screen cleaning mechanism of castor hulling is analyzed in this paper. And the numeric expression equations of the castor dynamics analysis are established. The correlation of floating speeds vp, air speeds w, the direction angle of airflow velocity $\beta$, the direction angle of vibration $\delta$, the friction angle $\varphi$, the slip coefficient, the fell coefficient and the jumping coefficient are analyzed.
\end{abstract}

\section{Introduction}

Castor is one of the world's top ten oil crops. The economic value of castor is very high, which is renewable green oil and can replace petroleum. There are many researches about agricultural materials hulling and cleaning structure, i.e. the effect of decorticating machine and the improving method [1], [2]. Existing researches primarily focuses on the mechanism of castor peeling and testing, the force analysis of castor in the process of shelling, the optimum design of key hulling parts and the peel rate and breakage rate testing [3].

However, there is few researches pay attention to the method and structure of castor cleaning except the aerodynamics analysis of air-and-screen cleaning mechanism of castor hulling [4]. In this paper, the relationships of floating speeds $v_{p}$, air speeds $w$, the direction angle of airflow velocity $\beta$, the direction angle of vibration $\delta$, the friction angle $\varphi$, the slip coefficient, the fell coefficient and the jumping coefficient are analyzed.

\section{Motion analysis of the shaker in the screening structure}

The shaker in the screening structure does harmonic reciprocating motion driven by a crank mechanism [5]. The schematic diagram is showed in Fig. 1.

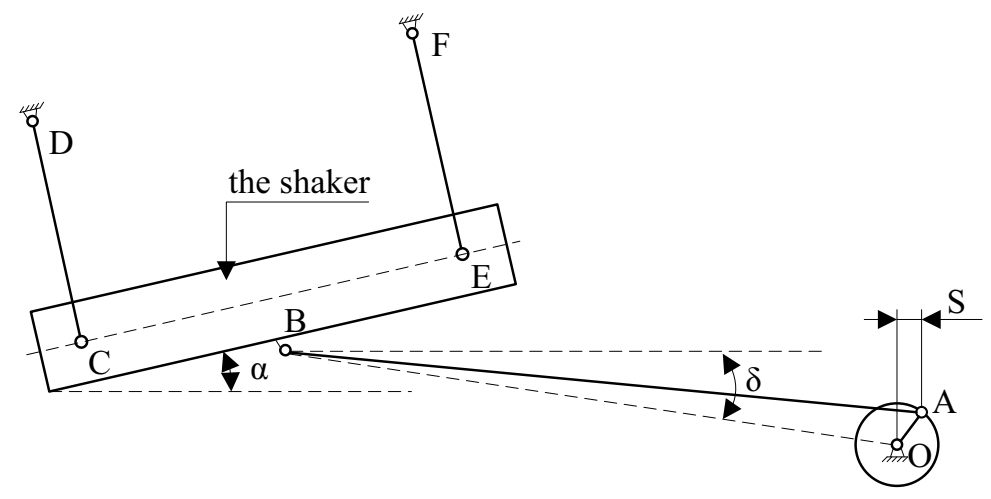

Figure 1. The motion schematic diagram of the shaker in the screening structure

The crank is $O A$. The linkage $C D$ is parallel and equal to the linkage $E F$. That the direction of the center of the crank $O$ connects with the hinge point $B$ in the shaker is the direction of vibration. The angle between it with the horizontal direction is the direction angle of vibration $\delta$. The direction angle of vibration is $0^{\circ}$. Compared with the crank $O A$, the length of the linkage $C D$, the linkage $E F$ and the linkage $O A$ is much longer. So, each point on the shaker is seen to do linear simple harmonic motion. Supposing the direction $O B$ is the positive direction of vibration, and the castor upstream direction along the screen surface is the positive direction of relative movement. Then, the equations of the screening structure motion relationship are as follow: 
Displacements: $S=R \cos \omega t$,

Speeds: $v=-R \omega \sin \omega t$,

Accelerations: $a=-\omega^{2} R \cos \omega t$.

where $R$ is the crank radius length, $\omega$ is the crank angular velocity, $t$ is the time.

\section{Dynamics analysis of the castor on the screen surface}

The motion of the castor on the screen surface is complex. In order to simplify the motion analysis, the castor is assumed that it was only affected by the friction of the shaker surface $F_{f}$, the force of the air flow $P$, the inertial force $Q$, the gravity $G$ and the supporting force $N$. the castor does the upward sliding, down sliding or jumping movement. The size and direction of the inertial force are determined by the size and direction of the acceleration of the castor. Therefore, before analyzing the state of motion of the castor on the shaker surface, the size and direction of it should be determined firstly. Then, the size parameters of the screening structure are selected.

\section{The upward sliding of the castor}

When the crank is located I or IV quadrant, $\omega t \in(0, \pi / 2) \bigcup(3 \pi / 2,2 \pi)$, the direction of acceleration $a$ is the positive direction which is a leftward direction. Since the inertial force direction is the opposite direction of the acceleration direction, so the inertial force is a rightward direction. Therefore, the castor along the shaker surface has an upward sliding tendency. The castor force analysis is showed in Fig.2.

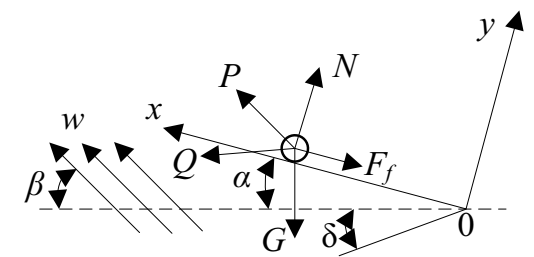

Figure 2. The castor upward sliding force analysis

The castor stress situation is as follows:

$$
\left\{\begin{array}{l}
m \mathrm{~d}^{2} x / \mathrm{d} t^{2}=Q \cos (\alpha+\delta)+P \cos (\beta-\alpha)-F_{f}-m g \sin \alpha \\
N+F \sin (\beta-\alpha)=m g \cos \alpha+Q \sin (\alpha+\delta) \\
F_{f}=f N=N \tan \varphi
\end{array}\right.
$$

where $Q=m R \omega^{2} \cos \omega t, P=m g w^{2} / v_{p}^{2}, \alpha$ is the shaker installation angle that is the angle between the horizontal direction and the shaker, $v_{p}$ is the floating speed of the castor, $w$ is air speeds, $\beta$ is the direction angle of airflow velocity, $\delta$ is the direction angle of vibration, $f$ is the coefficient of friction, $\varphi$ is the friction angle, $m$ is the mass of the castor particles and $g$ is the acceleration due to gravity.

Therefore,

$$
\frac{\mathrm{d}^{2} x}{\mathrm{~d} t^{2}}=\frac{R \omega^{2} \cos \omega t \cos (\alpha+\delta+\varphi)}{\cos \varphi}-g\left[\frac{\sin (\alpha+\varphi)}{\cos \varphi}-\frac{w^{2} \cos (\beta-\alpha-\varphi)}{v_{p}^{2} \cos \varphi}\right]
$$

When $R \omega^{2} \cos \omega t / g \geq \sin (\alpha+\varphi) / \cos (\alpha+\delta+\varphi)$, then $\mathrm{d}^{2} x / \mathrm{d} t^{2} \geq 0$, and the castor sliding upwards along the shaker surface. Since $0 \leq \cos \omega t \leq 1$, setting $\mathrm{d}^{2} x / \mathrm{d} t^{2}=0$, then the critical condition of the castor upward sliding is presented below:

$$
\frac{R \omega^{2}}{g} \cos \omega t=\frac{\sin (\alpha+\varphi)}{\cos (\alpha+\delta+\varphi)}-\frac{w^{2} \cos (\beta-\alpha-\varphi)}{v_{p}^{2} \cos (\alpha+\delta+\varphi)}
$$

So if the castor is demand to sliding upwards, the acceleration ratio $R \omega^{2} / g$ should satisfy conditions:

$$
\frac{R \omega^{2}}{g}>\frac{\sin (\alpha+\varphi)}{\cos (\alpha+\delta+\varphi)}-\frac{w^{2} \cos (\beta-\alpha-\varphi)}{v_{p}^{2} \cos (\alpha+\delta+\varphi)}
$$

Supposing the slip coefficient is $K_{1}$, $K_{1}=\frac{\sin (\alpha+\varphi)}{\cos (\alpha+\delta+\varphi)}-\frac{w^{2} \cos (\beta-\alpha-\varphi)}{v_{p}^{2} \cos (\alpha+\delta+\varphi)}$. Setting $K=R \omega^{2} / g$.

When $K>K_{1}$, then the castor sliding upwards along the shaker surface. Since $\omega=n \pi / 30$ ( $n$ is the number of turns of the crank revolutions per minute), the critical rotating speed of the crank when the castor sliding down along the shaker surface is as below:

$$
n_{1}=(30 / \pi) \sqrt{g K_{1} / R}
$$

If $-1 \leq \cos \omega t \leq 0$, then $K<K_{1}$, the castor sliding down.

\section{The down sliding of the castor}

When the crank is located II or III quadrant, $\omega t \in(\pi / 2,3 \pi / 2)$, the direction of acceleration $a$ is the negative direction which is a rightward direction. Since the inertial force direction is the opposite direction of the acceleration direction, so the inertial force is a leftward direction. Therefore, the castor has a down sliding tendency. The castor force analysis is showed in Fig. 3 .

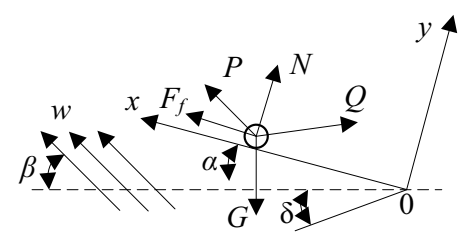

Figure 3. The castor down sliding force analysis 
The castor stress situation is as follows:

$\left\{\begin{array}{l}m \mathrm{~d}^{2} x / \mathrm{d} t^{2}=-Q \cos (\alpha+\delta)+P \cos (\beta-\alpha)+F_{f}-m g \sin \alpha \\ N+Q \sin (\alpha+\delta)+P \sin (\beta-\alpha)=m g \cos \alpha \\ F_{f}=f N=N \tan \varphi\end{array}\right.$

$\frac{\mathrm{d}^{2} x}{\mathrm{~d} t^{2}}=-\frac{R \omega^{2} \cos \omega t \cos (\alpha+\delta-\varphi)}{\cos \varphi}+g\left[\frac{\sin (\alpha-\varphi)}{\cos \varphi}-\frac{w^{2} \cos (\beta-\alpha-\varphi)}{v_{p}^{2} \cos \varphi}\right]$

Since $-1 \leq \cos \omega t \leq 0$, setting $\mathrm{d}^{2} x / \mathrm{d} t^{2}=0$, then the critical condition of the castor down sliding is presented below:

$$
\frac{R \omega^{2}}{g}>\frac{\sin (\alpha-\varphi)}{\cos (\alpha+\delta-\varphi)}+\frac{w^{2} \cos (\beta-\alpha-\varphi)}{v_{p}^{2} \cos (\alpha+\delta-\varphi)}
$$

Supposing the fell coefficient is $K_{2}$, $K_{2}=\frac{\sin (\alpha-\varphi)}{\cos (\alpha+\delta-\varphi)}+\frac{w^{2} \cos (\beta-\alpha-\varphi)}{v_{p}^{2} \cos (\alpha+\delta-\varphi)}$. When $K>K_{2}$, then the castor sliding down along the shaker surface.

\section{The jumping state of the castor}

When the crank is located I or IV quadrant, the direction of acceleration is the positive direction which is a rightward direction. It is in the opposite direction of the supporting force. Under the gravity, the pressure could not be 0 . Then, the castor is impossible to have jumping states when the crank is crank is located I or IV quadrant.

When the crank is located II or III quadrant, the inertial force $Q$ equals $m R \omega^{2} \cos \omega t$. The direction of it is the same as the direction of the supporting force. So, the castor has a tendency to be thrown upward. Then, when the supporting force $N$ is 0 , the castor would be thrown from the shaker surface.

Therefore,

$$
N=m g \cos \alpha+Q \sin (\alpha+\delta)-P \sin (\beta-\alpha)=0
$$

$m g \cos \alpha-m R \omega^{2}|\cos \omega t| \sin (\alpha+\delta)-m g w^{2} \sin (\beta-\alpha) / v_{p}^{2}=0$

where $|\cos \omega t| \leq 1$.

So, the critical condition of the castor jumping is presented below:

$$
K=\frac{R \omega^{2}}{g}>\left(\frac{\cos \alpha}{\sin (\alpha+\delta)}-\frac{w^{2} \sin (\beta-\alpha)}{v_{p}^{2} \cos (\alpha+\delta)}\right)
$$

Supposing the jumping coefficient is $K_{3}$, $K_{3}=\left(\frac{\cos \alpha}{\sin (\alpha+\delta)}-\frac{w^{2} \sin (\beta-\alpha)}{v_{p}^{2} \cos (\alpha+\delta)}\right)$. When $K>K_{3}$, then the castor is in jumping states. Then, the rotating speed of the crank can be calculated as follows:

$$
n_{3}=(30 / \pi) \sqrt{g K_{3} / R}
$$

From the above analysis, if friction angle $\varphi$, the shaker installation angle $\alpha$, the direction angle of airflow $\beta$ and the floating speed of the castor $v_{p}$ can be determined, the critical air speeds $w$ and the critical crank angular velocity $\omega$ could be calculated.

The states of the castor are depends on the relationship of coefficient $K, K_{1}, K_{2}$, and $K_{3}$. The states are analyzed as follows:

(1) If $K \leq K_{1}$ and $K \leq K_{2}$, the castor is in a relatively quiescent state.

(2) If $K_{2}>K>K_{1}$, the castor only have the upward sliding state.

(3) If $K_{1}>K>K_{2}$, the castor only have the down sliding state.

(4) If $K>K_{1}>K_{2}$, the castor have both upward and down sliding states. But more castors have an upward sliding tendency.

(5) If $K>K_{2}>K_{1}$, more castors have a down sliding tendency.

(6) If $K>K_{3}$, the castor is in jumping states.

\section{Conclusion}

In air-and-screen cleaning mechanism, the castor motion on the shaker is analyzed and the numeric expression equations of the castor motion are established. The relationships of the castor states with coefficient $K, K_{1}$, $K_{2}$, and $K_{3}$ are analyzed. In the future, the association curves of floating speeds $v_{p}$, air speeds $w$, the direction angle of airflow $\beta$, the direction angle of vibration $\delta$, the friction angle $\varphi$, the coefficient $K, K_{1}, K_{2}$, and $K_{3}$ would be analyzed. The main factors that affect the cleaning rate and loss rate of mechanism would be identified.

\section{Acknowledgements}

The work was supported by the Science \& Technology Foundation of Guangdong Province (No.2013B020313004).

\section{References}

1. L. Zhang, L. Gong, Status quo about shelling machine and discussing about improving methods, Food and Machinery, 22 (4), pp. 72-74, (2006)

2. C. Deng, D. Tao, Y. Gao, Research situation and development trend on grain sorting machinery, Journal of Agricultural Mechanization Research, 2, pp. 5-7, (2005)

3. Y. Cao, C. Li, Z. Zhang, Y. Qing, L. Yao, Improvement design and test to key components of castor capsule hulling device, 28(18), pp. 16-22, (2015)

4. L. Yao, Y. Cao, C. Li, Z. Fang, Dynamics property of air-and-screen cleaning mechanism of castor hulling, 12, pp. 70-74, (2014) 
5. C. Chen, Z. Wang, Q. Li, Analysis of aerodynamic properties of rape extractions, Transactions of the Chinese Society for Agricultural Machinery, 35(5), p. 90-93, (2004) 